\title{
Editorial
}

\section{The State of Aortic Surgery in India}

\author{
Mohammed Idhrees ${ }^{1 *}$, Mohammed Ayyub², Bashi V. Velayudhan' \\ 'Institute of Cardiac and Aortic Disorders (ICAD), SRM Institutes for Medical Science (SIMS Hospital), Vadapalani, Chennai, India. \\ ${ }^{2}$ Dhanalakshmi Srinivasan Medical College \& Hospital, Perambalur, Tamil Nadu, Chennai, India
}

*Address for Correspondence: Dr. A. Mohammed Idhrees MCh, FAIS, Cardio Thoracic and Vascular Surgeon, Institute for Cardiac and Aortic Disorders, SRM Institute for Medical Sciences (SIMS Hospitals), Chennai, India. E mail: a.m.idhrees@gmail. com

Received: 22 August 2020; Accepted: 05 October 2020; Published: 08 October 2020

Citation of this article: Idhrees M, Ayyub M, Velayudhan BV (2020) The State of Aortic Surgery in India. Rea Int J of Card and Cardio Med. 1(1): 008-008. DOI: 10.37179/rijccm.000003.

Copyright: (C) 2020 Idhrees AM, et al. This is an open access article distributed under the Creative Commons Attribution License, which permits unrestricted use, distribution, and reproduction in any medium, provided the original work is properly cited.

\section{Introduction}

Aortic diseases, to a great extent, are a problem of the elderly. In the last 50 years, the life expectancy of an Indian has increased by 30 years. The technical developments have grown multi-folds, helping the diagnosis of patients at an early stage. The above in combination with lifestyle, diabetes, and increase in population has placed the country with a high incidence of cardiovascular and aortic disease. Aortic diseases include aortic dissections and aortic aneurysms. These can be catastrophic when not treated at the right time. If the patient is not treated, the mortality rate is $50 \%$ at 24 hours. At present a very few centres in India are performing complex aortic surgeries and providing emergency aortic services.

\section{Challenges in India}

Certain vascular grafts and endovascular stents are not available in India. Unlike many western countries, the cost of the procedures falls on the shoulder of the patients themself. With a literacy rate close to $75 \%$ and the Gross national Income per capita $\$ 7060$ (UK: Literacy rate is $99 \%$ and Gross national Income per capita is $\$ 43160$ ), most of the patients present to the doctor at a late stage of disease. These patients have to accept a double burden, wherein they have to pay for the procedure and have loss of pay during hospital admission. In case of emergency, the patients have few questions to ask him/her before deciding for treatment. Where to go to get treated? How to reach the centre to get operated? How much will be the cost?
The challenges in multifield which includes

1. Expertise: Not many surgeons are trained in complex aortic surgeries at present in the country

2. Transport: India extends close to $3000 \mathrm{~km}$ from north to south and east to west. Transporting a patient in case of emergency from the rural India to places where surgery is done is a true challenge.

3. Lack of awareness: This included the risk factors and the medical conditions related to the aorta among the general population.

\section{Overcoming Challenges}

With so many stumbling blocks, we need to perform to continue performing the surgery. To negate the non-availability of grafts and hybrid operating room, we customise our surgery, operating room and materials to suit the need of our people - "The 'Indigenous Way". This has helped us to become more innovative wherein we have described new techniques in International journals in field of aortic surgery.

To help the needy, we have formed a charitable organisation, which receives funds from Indian and many foreign nationalities. But it is a drop in the ocean to help the large number of patients who need aortic surgery. We have a reportedly over 300000 patients in a year suffering from aortic diseases in our country with a handful of centres performing aortic surgery. 